\title{
Mid-infrared photonics devices in SOI
}

\author{
G. Z. Mashanovich ${ }^{\mathrm{a}}$, M. Nedeljkovic ${ }^{\mathrm{a}}$, M. M. Miloševićb, Y. Hu ${ }^{\mathrm{a}}$, T. M. Ben Masaud ${ }^{\mathrm{c}}$,

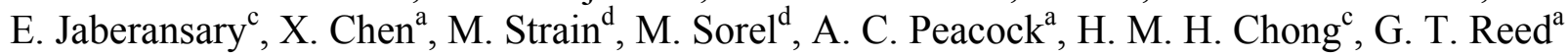 \\ ${ }^{a}$ Optoelectronics Research Centre, University of Southampton, Southampton, SO17 1BJ, UK \\ ${ }^{b}$ Department of Electronics Engineering, University of Surrey, Guildford, GU2 7XH, UK \\ ${ }^{\mathrm{c}}$ Electronics and Computer Science, University of Southampton, Southampton, SO17 1BJ, UK \\ ${ }^{\mathrm{d}}$ School of Engineering, University of Glasgow, Glasgow G12 8LT, UK
}

\begin{abstract}
In this paper we present silicon photonics devices designed for the 3-4 $\mu \mathrm{m}$ wavelength region including waveguides, MMIs, ring resonators and Mach-Zehnder interferometers. The devices are based on silicon on insulator (SOI) platform. We show that 400-500 $\mathrm{nm}$ high silicon waveguides can have propagation losses as low as $\sim 4 \mathrm{~dB} / \mathrm{cm}$ at $3.8 \mu \mathrm{m}$. We also demonstrate MMIs with insertion loss of $0.25 \mathrm{~dB}$, high extinction ratio asymmetric Mach-Zehnder interferometers, and SOI ring resonators. This combined with our previous results reported at $3.4 \mu \mathrm{m}$ confirm that SOI is a viable platform for the 3-4 $\mu \mathrm{m}$ region and that low loss mid-infrared passive devices can be realized on it. Keywords: Mid-infrared, silicon, silicon-on-insulator, Mach-Zehnder interferometer, multimode interference, ring resonator.
\end{abstract}

\section{INTRODUCTION}

The mid-infrared (mid-IR, 3-20 $\mu \mathrm{m}$ ) offers applications in diverse areas due to strong absorption bands of many chemical and biological molecules throughout this wavelength range. Applications might be found in medicine, environmental sensing and pollution monitoring, industrial process control, and toxic gas or chemical detection for defence and security. This has recently driven interest in mid-IR group-IV photonics, which offers a potential route to compact, portable sensors in which photonic and electronic functions are integrated on a single chip [1].

Most such sensors would rely on a library of integrated photonic components such as waveguides, splitters, couplers, filters, interferometers, modulators, sources and detectors, among others. For near-infrared (NIR) silicon photonics the design and fabrication of most of these components is now well explored. However, in moving towards longer wavelengths there are new challenges that must be overcome, of which the first is that silicon-on-insulator (SOI), the most commonly used material platform for NIR silicon photonics, has $\mathrm{SiO}_{2}$ as the cladding material, which begins to absorb heavily for $\lambda>3.6 \mu \mathrm{m}$. Transmission has been reported through a number of alternative waveguide platforms to SOI at different wavelengths through the mid-IR, such as silicon-on-porous silicon [2], silicon-on-sapphire [3, 4], silicon membrane [5, 6], and germanium-on-silicon [7]. However, as SOI is now so well understood for photonics and is readily available, wherever it is possible to use the material it will likely be the platform of choice.

In our work we explore the transmission properties of SOI waveguides throughout the $3-4 \mu \mathrm{m}$ range in order to establish the propagation loss of different SOI waveguide configurations at these wavelengths, and to demonstrate passive components, including multimode interferometers (MMIs), ring resonators and Mach-Zehnder interferometers (MZIs) in SOI material. We also discuss here how the design of some of these devices varies with wavelength.

\section{MID-INFRARED CHARACTERISATION SETUP}

Our mid-IR characterization setup has been described in detail elsewhere [2]. A HeNe source at $3.39 \mu \mathrm{m}$ with $2 \mathrm{~mW}$ optical power and a Quantum Cascade Laser that is tunable from 3.72-3.90 $\mu \mathrm{m}$ with a maximum power of $150 \mathrm{~mW}$ have been used. Light is coupled into a fluoride based mid-IR fibre using a $\mathrm{ZnSe}$ lens, and these fibers are used for butt coupling to and from the chip. When light from a NIR broadband source is first launched into the fibre, in combination with a NIR camera it is used to confirm that light is being coupled into the waveguide. A liquid nitrogen cooled InSb detector is used for detection of mid-IR light. In each of the measurements described in this paper, only TE light is coupled into the fibres, and we find that polarization change in the fibre is minimal. Both the HeNe and QCL lasers emit 
linearly polarized light, and we use a half-wave plate and polarizers that are suitable for this wavelength range for additional polarization control.

\section{SOI WAVEGUIDES}

For all of the waveguide types presented here, the propagation loss was measured by the cut-back method, where waveguides of different lengths are fabricated on the same chip, and the loss is measured from the relationship between waveguide length and optical transmission. Each chip was prepared for measurement by polishing of the chip facets by abrasive discs, to ensure sufficiently smooth waveguide facets.

\section{1. $2 \mu \mathrm{m}$ height rib waveguides}

We first reported propagation loss measurements of SOI rib waveguides at $\lambda=3.39 \mu \mathrm{m}$, with dimensions width (W) $\times$ height $(\mathrm{H})=2 \mu \mathrm{m} \times 2 \mu \mathrm{m}$ and etch depth $=1.2 \mu \mathrm{m}$, with a $2 \mu \mathrm{m}$ BOX thickness [2]. The waveguides were fabricated by UV lithograhy and RIE etching. Their propagation loss was measured to be $0.6 \pm 0.2 \mathrm{~dB} / \mathrm{cm}$. More recently we have published propagation loss results for these same waveguides at the wavelengths $3.73 \mu \mathrm{m}$ and $3.80 \mu \mathrm{m}$, for which the losses were measured to be $1.5 \pm 0.2 \mathrm{~dB} / \mathrm{cm}$ and $1.8 \pm 0.2 \mathrm{~dB} / \mathrm{cm}$ respectively [8]. The loss values given here are the losses measured after the waveguide roughness was reduced by thermal oxidation, which lowered the propagation loss. We note that the propagation loss increases quite significantly even over this wavelength range. Part of this increase can be accounted for by increased optical mode interaction with sidewall roughness, though the remainder reflects the increased absorption in $\mathrm{SiO}_{2}$ for $\lambda>3.6 \mu \mathrm{m}$.

\subsection{Submicron strip and rib waveguides}

Strip waveguides with smaller dimensions are able to have low loss for much smaller bend radii than larger rib waveguides, hence tighter integration of components on chip for a smaller device footprint is possible. However, because of greater interaction of the optical mode with both the sidewalls and the BOX layer, there is larger propagation loss. We have fabricated and characterised SOI strip waveguides with $\mathrm{H}=500 \mathrm{~nm}$, a $3 \mu \mathrm{m}$ BOX layer thickness and width variations of $1.0 \mu \mathrm{m}, 1.2 \mu \mathrm{m}$ and $1.4 \mu \mathrm{m}$, for which we have reported the results in [9]. The waveguides were fabricated by e-beam lithography and ICP etching, and were treated by thermal oxidation after lithography, which produced a 20-30nm thick layer of thermal oxide over the waveguides. Simulations suggested that the propagation loss would decrease as the width of the waveguides was increased, and this was confirmed by the experimental measurements. The simulated losses were $6.6 \mathrm{~dB} / \mathrm{cm}, 5.1 \mathrm{~dB} / \mathrm{cm}$ and $3.5 \mathrm{~dB} / \mathrm{cm}$, while measured losses were $5.9 \pm 0.4 \mathrm{~dB} / \mathrm{cm}, 5.2 \pm 1.6 \mathrm{~dB} / \mathrm{cm}$ and $4.6 \pm 1.1 \mathrm{~dB} / \mathrm{cm}$ for W $=1.0 \mu \mathrm{m}, 1.2 \mu \mathrm{m}$ and $1.4 \mu \mathrm{m}$ respectively.

We have also fabricated $400 \mathrm{~nm}$ high SOI waveguides with $350 \mathrm{~nm}$ etch depth, $1.1 \mu \mathrm{m}$ width and $2 \mu \mathrm{m}$ BOX thickness. These waveguides were also fabricated by e-beam lithography and ICP etching, though they have no thermal oxide layer. The measured propagation loss was $9.0 \mathrm{~dB} / \mathrm{cm}$, which is similar to the losses of the $500 \mathrm{~nm}$ high strip waveguides before roughness reduction. We expect that thermal oxidation of these waveguides and increasing their width would reduce their losses to similar values to those measured for 500nm high waveguides.

\section{PASSIVE DEVICES}

The passive devices for which the results are presented in this section make use of the waveguides with submicron Si layer thickness; they are based either on strip waveguides with 500nm Si height or rib waveguides with 400nm Si height. The fabrication methods in each case are the same as those used for waveguides, although thermal oxidation has not been used to reduce the losses of these structures, except where stated.

\section{1. $1 \times 2$ Multimode interferometers}

Multimode interferometers are devices that work on the principal of self-imaging in a wide multimode waveguide, and are used as splitters or couplers for different numbers of input and output waveguides. We have demonstrated MMIs with one input and two outputs, that have tapered input and output ports to reduce the insertion loss into the devices, as was demonstrated by Thomson et al [10]. Fig. 1 shows an SEM image of one of our fabricated MMIs, with the design variables marked on the image. 


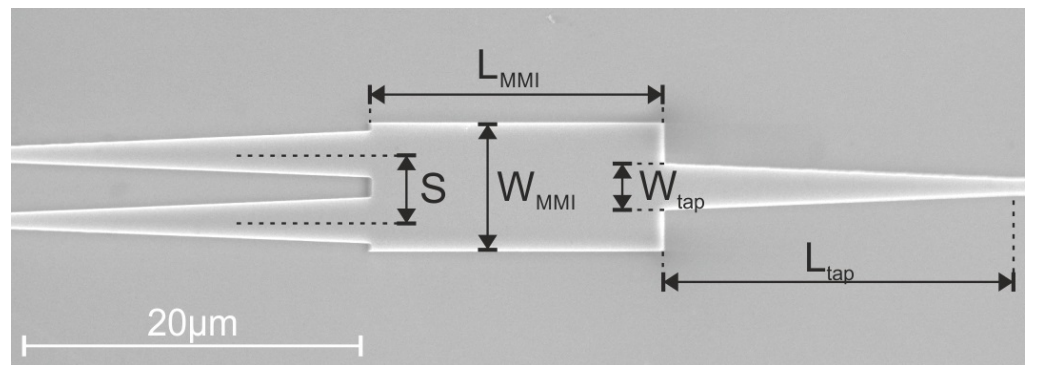

Figure 1. SEM image of fabricated $1 \times 2$ MMI, with tapered input and output ports. Design variables are marked on the image.

Soldano [11] gave the locations of the self-imaging points of a $1 \times 2 \mathrm{MMI}$ along its length, $z$, and the location at which two-fold self-imaging occurs, in a $2 \mathrm{D}$ approximation, is:

$$
z=\frac{n_{e f f} W_{e f f}^{2}}{2 \lambda},
$$

where $n_{\text {eff }}$ is the effective refractive index of the waveguide and $W_{\text {eff }}$ is the effective width of the MMI, as defined in [12]. From this equation we see that for a given device width, the length of the device is inversely proprtional to the wavelength, so devices will have a smaller footprint at longer wavelengths. This equation was used for an initial estimate of the device length, followed by detailed 3D simulation of the device using the Photon Design Fimmprop software package to refine the design.

Devices were fabricated that were based on both the $500 \mathrm{~nm}$ strip waveguide design and the $400 \mathrm{~nm}$ rib waveguide design. For the device with $500 \mathrm{~nm}$ Si layer thickness, the designed dimensions were $\mathrm{W}_{\mathrm{MMI}}=8 \mu \mathrm{m}, \mathrm{L}_{\mathrm{MMI}}=21.83 \mu \mathrm{m}, \mathrm{W}_{\text {tap }}$ $=3.0 \mu \mathrm{m}, \mathrm{L}_{\text {tap }}=20 \mu \mathrm{m}$ and $\mathrm{S}=4.0 \mu \mathrm{m}$, with simulations predicting an insertion loss of $0.11 \mathrm{~dB}$. For the device with $400 \mathrm{~nm}$ Si layer thickness, the designed dimensions were $\mathrm{W}_{\mathrm{MMI}}=8 \mu \mathrm{m}, \mathrm{L}_{\mathrm{MMI}}=21.0 \mu \mathrm{m}, \mathrm{W}_{\text {tap }}=2.6 \mu \mathrm{m}, \mathrm{L}_{\text {tap }}=20 \mu \mathrm{m}$ and $\mathrm{S}$ $=4.18 \mu \mathrm{m}$, with simulations predicting an insertion loss of $0.08 \mathrm{~dB}$. The insertion loss of fabricated devices was measured by chaining different numbers of pairs of MMIs together and measuring the transmission through them. Initial fabrication of MMIs with $500 \mathrm{~nm}$ SOI was reported in [9] as having an insertion loss of $3.6 \pm 0.2 \mathrm{~dB} / \mathrm{MMI}$ at $\lambda=3.74 \mu \mathrm{m}$. This was much higher than expected and was attributed to non-optimised dimensions and higher sidewall roughness. A second fabrication run, where the fabricated devices corresponded well to design dimensions gave a much smaller loss of $0.29 \pm 0.17 \mathrm{~dB}$. The devices with $400 \mathrm{~nm}$ thick Si layer exhibited insertion loss of $0.25 \pm 0.02 \mathrm{~dB} / \mathrm{MMI}$. Fig. 2 shows the chart of transmission plotted against number of MMIs from which the loss figure was calculated [13]. The loss figures for both of these devices rival the best achieved loss figures for NIR silicon photonics [10, 14], and are a significant advance over those achieved so far in the mid-IR $[6,9]$.

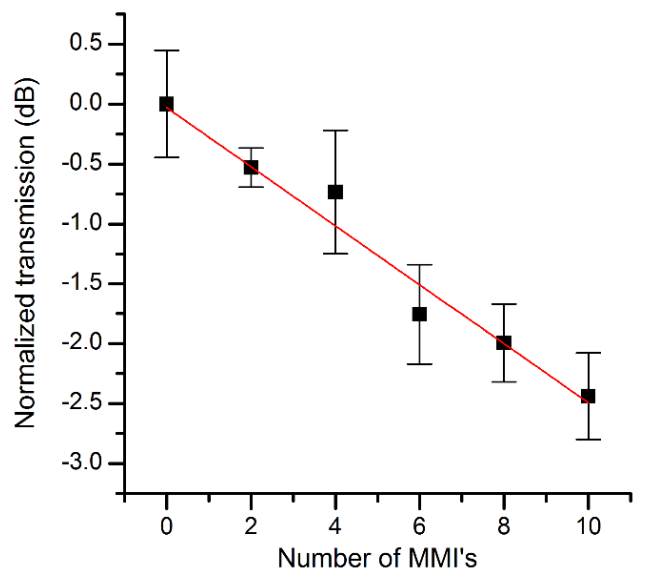

Figure 2. Number of MMIs vs. normalized transmission for MMI's with $\mathrm{H}=400 \mathrm{~nm}, \mathrm{D}=350 \mathrm{~nm}, \mathrm{~W}_{\mathrm{MMI}}=8 \mu \mathrm{m}, \mathrm{L}_{\mathrm{MMI}}=21.0 \mu \mathrm{m}$, $\mathrm{W}_{\text {tap }}=2.6 \mu \mathrm{m}, \mathrm{L}_{\text {tap }}=20 \mu \mathrm{m}$ and $\mathrm{S}=4.18 \mu \mathrm{m}$. The slope of the graph gives the insertion loss $/ \mathrm{MMI}$ of $0.25 \pm 0.02 \mathrm{~dB} / \mathrm{MMI}[13]$. 


\subsection{Racetrack and ring resonators}

Ring resonators are used as wavelength filters and for converting a phase change into an amplitude change in either modulators or sensors. We have demonstrated racetrack resonators based on $500 \mathrm{~nm}$ high strip waveguides [9]. The devices had a bending radius of $100 \mu \mathrm{m}$, a coupling region length of $50 \mu \mathrm{m}$, and an edge to edge spacing between the bus waveguide and ring waveguide of $1.2 \mu \mathrm{m}$. They were treated by thermal oxidation to reduce surface roughness. Fig. 3 shows an SEM image of one of the fabricated resonators.

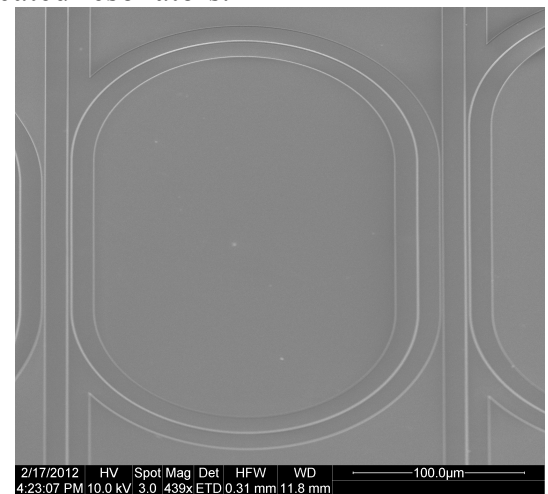

Figure 3. An SEM image of a racetrack ring resonator in SOI material with 500nm Si thickness [9]. The bending radius is $100 \mu \mathrm{m}$, coupling region length is $50 \mu \mathrm{m}$ and ring-bus waveguide edge to edge separation is $1.2 \mu \mathrm{m}$.

For filters an important property is their free spectral range (FSR), which for a ring/racetrack resonator is given in [12] as:

$$
F S R=\frac{\lambda_{r}^{2}}{n_{g} d},
$$

where $\lambda_{r}$ is the wavelength of the nearest resonant peak, $n_{g}$ is the group index of refraction of the waveguide, and $d$ is the length of the waveguide that makes up the ring/racetrack. In moving to longer wavelengths, the length of the ring waveguide will need to increase proportionally to the square of the wavelength to maintain a particular FSR.

The transmission spectrum of the racetrack resonator was measured using the tunable QCL. The FSR of the device was measured to be $4.12 \mathrm{~nm}$ with an associated group index of 4.64 , compared to theoretical values of FSR $=4.24 \mathrm{~nm}$, and $n_{g}$ $=4.53$. The experimentally measured resonance peaks exhibited an extinction ratio up to $10 \mathrm{~dB}$, and an average Q-factor of 8.2k. From simulations the Q-factor was expected to be in the 30-40k range. This discrepancy might arise due to the QCL having mode-hopping behaviour, which could reduce the effective linewidth of the measurement, and be reflected in a lower Q-factor value.

\subsection{Mach-Zehnder interferometers}

Mach-Zehnder interferometers for the mid-IR have been reported in the literature in [15] for use in a group index measurement of photonic crystals, and in [16] as part of an optical modulator in silicon. Neither paper investigated the characteristics of the MZI itself, so we report here the extinction ratios and insertion losses of asymmetric MZIs based on both $400 \mathrm{~nm}$ and $500 \mathrm{~nm}$ high $\mathrm{Si}$ waveguides that have one input and one output, and that use the MMIs reported above as splitters and couplers.

Like ring resonators, Mach-Zehnder interferometers can be used as wavelength filters, or for converting a refractive index change into a change in optical transmission. The advantage of MZIs is that they are far less sensitive to thermal variations than rings, though they also typically have a much larger device footprint. The FSR of an asymmetric $1 \times 1$ MZI is given by:

$$
F S R=\frac{\lambda^{2}}{n_{g} \Delta L-\lambda}
$$

where $\Delta L$ is the arm length difference [13]. The MZI with $400 \mathrm{~nm}$ Si height waveguides had $\Delta L=300 \mu \mathrm{m}$, and the MZI with $500 \mathrm{~nm}$ high waveguides had $\Delta L=350 \mu \mathrm{m}$. The transmission spectrum of the $400 \mathrm{~nm}$ high MZI is shown in Figure 4, and that of the $500 \mathrm{~nm}$ high MZI is shown in Figure 5. 


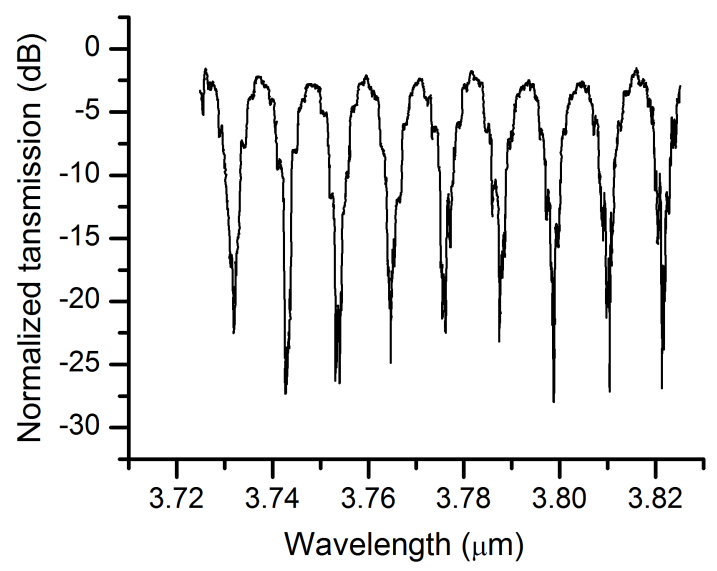

Figure 4. Normalized transmission spectrum of an MZI in SOI based on 400nm high rib waveguides with 350nm etch depth [13].

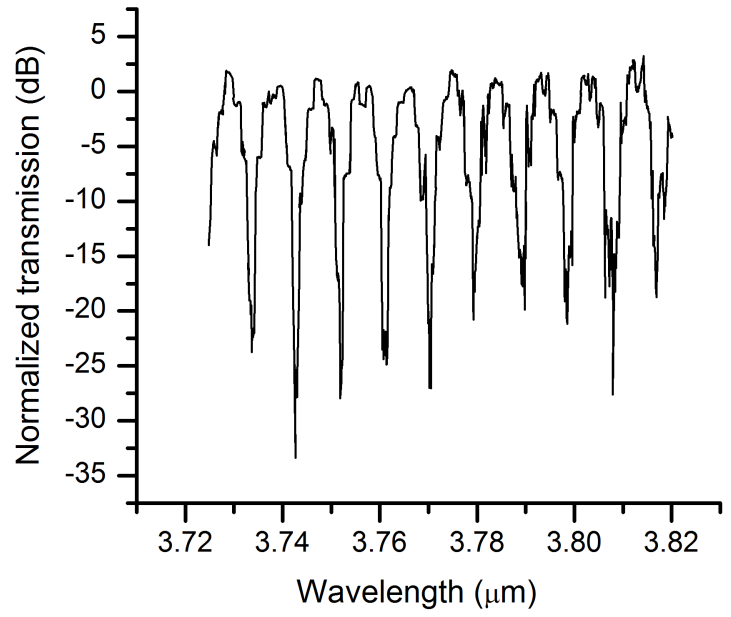

Figure 5. Normalized transmission spectrum of an MZI in SOI based on 500nm high strip waveguides [13].

The MZI based on 400nm high waveguides exhibited an extinction ratio of up to $27 \mathrm{~dB}$ and insertion loss of $1.6-2.4 \mathrm{~dB}$, while for the device based on 500nm high waveguides the extinction ratio of up to $34 \mathrm{~dB}$ was measured with the insertion loss being too small to measure due to noise in the spectra of the MZI and of normalization waveguides.

\section{SUMMARY}

We have investigated silicon on insulator as a material platform for integrated photonics in the 3-4 $\mu$ m wavelength region by fabricating rib and strip waveguides, and other passive devices, and testing them at wavelengths through this range. Rib waveguides with $2 \mu \mathrm{m}$ Si device layer thickness had losses as low as $0.6-0.7 \mathrm{~dB} / \mathrm{cm}$ at $3.39 \mu \mathrm{m}$, and increasing to $1.8 \mathrm{~dB} / \mathrm{cm}$ at $3.80 \mu \mathrm{m}$. Strip waveguides with $500 \mathrm{~nm}$ height had propagation loss as low as $4.6 \pm 1.1 \mathrm{~dB} / \mathrm{cm}$ at $\lambda=3.74 \mu \mathrm{m}$. These results show that SOI can be a very useful material platform at wavelengths up to almost $4 \mu \mathrm{m}$.

We have demonstrated multimode interferometers, racetrack and ring resonators and Mach-Zehnder interferometers based on submicron strip and rib waveguides. MMIs exhibited very low insertion losses of only $0.25 \pm 0.02 \mathrm{~dB} / \mathrm{MMI}$, which approaches the best reported figures in near-infrared silicon photonics. The tested racetrack resonators had extinction ratios of up to $10 \mathrm{~dB}$, and a Q-factor of $8.2 \mathrm{k}$. The MZIs had extinction ratios of up to $34 \mathrm{~dB}$, and negligible insertion loss.

The fabricated passive devices would likely be very important components of any integrated photonic system, for example for sensing, and confirm that the design methods that are used for NIR silicon photonics are transferable to wavelengths in the mid-infrared. 


\section{ACKNOWLEDGEMENTS}

This work is supported by the Royal Society (G. Mashanovich's URF "Mid-infrared Silicon Photonics") and by EPSRC through "UK Silicon Photonics" programme.

\section{REFERENCES}

[1] R.A. Soref, "Mid-infrared photonics in silicon and germanium," Nature Photonics 4, 495-497 (2010).

[2] G. Z. Mashanovich, M. M. Milosevic, M. Nedeljkovic, N. Owens, B. Xiong, E. J. Teo, and Y. Hu, "Low loss silicon waveguides for the mid-infrared," Opt. Express 19(8), 7112-7119 (2011).

[3] T. Baehr-Jones, A. Spott, R. Ilic, B. Penkov, W. Asher, and M. Hochberg, "Silicon-on-sapphire integrated waveguides for the mid-infrared," Opt. Express 18(12), 12127-12135 (2010).

[4] F. Li, S. Jackson, C. Grillet, E. Magi, D. Hudson, S. J. Madden, Y. Moghe, C. O’Brien, A. Read, S. G. Duvall, P. Atanackovic, B. J. Eggleton and D. Moss, "Low propagation loss silicon-on-sapphire waveguides for the midinfrared," Opt. Express 19(16), 15212-15220 (2011).

[5] Z. Cheng, X. Chen, C. Y. Wong, K. Xu, and H. K. Tsang, "Mid-infrared suspended membrane waveguide and ring resonator on silicon-on-insulator," IEEE Phot. J. 4, 1510-1519 (2012).

[6] Y. Wei, G. Li, Y. Hao, Y. Li, J. Yang, M. Wang and X. Jiang, "Long-wave infrared 1 x 2 MMI based on air-gap beneath silicon rib waveguides," Opt. Express, 19(17), 15803-15809 (2011).

[7] Y-C Chang, V. Paeder, L. Hvozdara, J-M Hartmann, and H.P. Herzig, "Low-loss germanium strip waveguides on silicon for the mid-infrared," Opt. Letters 37, 2883-2885 (2012).

[8] M. M. Milošević, D. J. Thomson, X. Chen, D. Cox, and G. Z. Mashanovich, "Silicon waveguides for the 3-4 $\mu \mathrm{m}$ wavelength range," 8th IEEE Group IV Photonics conference, London, UK, (2011).

[9] M. M. Milosevic, M. Nedeljkovic, T.-B. Masaud, E. Jaberansary, H. M. H. Chong, N. G. Emerson, G. T. Reed, and G. Z. Mashanovich, "Silicon waveguides and devices for the mid-infrared," Appl. Phys. Lett. 101, 121105 (2012).

[10] D. J. Thomson, Y. Hu, G. T. Reed, and J.-M. Fedeli, "Low loss MMI couplers for high performance MZI modulators," IEEE Photon. Technol. Lett. 22, 1485-1487 (2010).

[11] L. B. Soldano and E. C. M. Pennings, "Optical multi-mode interference devices based on self-imaging: principles and applications," J. Lightwave Technol. 13, 615-627 (1995).

[12] G. T. Reed, Silicon photonics: the state of the art (John Wiley \& Sons, Ltd, 2008).

[13] M. Nedeljkovic, T. B. Masaud, M. Strain, M. Sorel, M. M. Milošević, N. G. Emerson, G. T. Reed, H. M. H. Chong, and G. Z. Mashanovich, "Mid-infrared Mach-Zehnder and multimode interferometers in SOI," (submitted).

[14] W. Bogaerts, S. K. Selvaraja, P. Dumon, J. Brouckaert, K. De Vos, D. Van Thourhout, and R. Baets, "Silicon-oninsulator spectral filters fabricated with CMOS technology," IEEE J. Sel. Top. Quantum Electron. 16, 33-44 (2010).

[15] C. Reimer, M. Nedeljkovic, D. J. M. Stothard, M. O. S. Esnault, C. Reardon, L. O’Faolain, M. Dunn, G. Z. Mashanovich, and T. F. Krauss, "Mid-infrared photonic crystal waveguides in silicon," Opt. Express 20, 2936129368 (2012).

[16] M. A. Van Camp, S. Assefa, D. M. Gill, T. Barwicz, S. M. Shank, P. M. Rice, T. Topuria, and W. J. Green, "Demonstration of electrooptic modulation at $2165 \mathrm{~nm}$ using a silicon Mach-Zehnder interferometer," Opt. Express 20, 28009-28016 (2012). 